\title{
Cuestionario de apoderados de estudiantes haitianos sobre prácticas de alfabetización y lenguas
}

\author{
Questionnaire of representatives of Haitian students about literacy \\ and language practices
}

\author{
Gloria Toledo Vega ${ }^{a}$, Francisco Quilodrán ${ }^{b}$, Alejandra Meneses ${ }^{c}$ \\ ${ }^{a}$ Facultad de Letras, Pontificia Universidad Católica de Chile. \\ gtoledo@uc.cl \\ ${ }^{b}$ Programa de Español, Pontificia Universidad Católica de Chile. \\ fgquilodran@uc.cl \\ ${ }^{c}$ Facultad de Educación, Pontificia Universidad Católica de Chile. \\ alejandra.meneses@gmail.com
}

\begin{abstract}
La percepción de los apoderados sobre las prácticas de alfabetización de sus hijos en la escuela es un tema relevante, especialmente para los apoderados inmigrantes. Conocer sus percepciones ayuda a identificar los desafíos que pueden tener los estudiantes y sus familias en el proceso de inmersión y aprendizaje del español como lengua extranjera (ELE). Para esto, diseñamos y validamos un cuestionario sobre la percepción de apoderados inmigrantes haitianos acerca de la relación de sus hijos con el español, el creole y el francés; su motivación hacia el ELE; y la relación de los apoderados con el colegio en tanto contexto de aprendizaje del español. La validación se realizó con 132 apoderados haitianos de escuelas municipales de la Región Metropolitana. Se realizaron análisis psicométricos para una confiabilidad aceptable del instrumento y se determinó la existencia de las tres dimensiones que conforman el constructo medido: uso de lenguas, motivación y relación con la escuela.
\end{abstract}

Palabras claves: Validación, percepciones, inmigrantes, español como lengua extranjera, escuela chilena.

\begin{abstract}
The parents' perception of literacy practices of their children in the school is a relevant issue, especially in the case of immigrant parents. Knowing their perceptions helps to identify the challenges that students and their families may have in the process of immersing and learning Spanish as a foreign language (SFL). For this purpose, we designed and validated a questionnaire about the perception of immigrant Haitian parents about the relationship of their children with Spanish, Creole, and French; their motivation towards SFL; and the relationship of the parents with the school as a context to learn Spanish. The validation was carried out with 132 Haitian participants from municipal schools in the Metropolitan Region. Psychometric analyzes were performed for an acceptable reliability of the instrument, and the existence of the three dimensions that make up the measured construct were determined: use of languages, motivation and relationship with the school.
\end{abstract}

Key words: Validation, perceptions, immigrants, Spanish as Foreign Language, Chilean school. 


\section{INTRODUCCIÓN}

El fenómeno migratorio en Chile ha centrado gran parte de su atención en la comunidad haitiana. Las causas de esto pueden relacionarse con el aumento explosivo de estos ciudadanos -al comparar entre el 2010 y el 2016 hubo un aumento del $4.500 \%$ en la entrega de permanencias definitivas (Departamento de Extranjería y Migración, 2018)- y al hecho de ser una comunidad con una etnia y un lenguaje diferente al chileno. Hasta el momento, las investigaciones que se han realizado en Chile sobre este grupo se han enfocado en temas descriptivos (Pérez, 2016; Riedemann y Stefoni, 2015; Valenzuela et al., 2014, Stefoni et al., 2008). Hasta hoy no se han realizado estudios para comprender cómo los apoderados inmigrantes perciben la relación que tienen sus hijos con la lengua y la escuela de acogida. Conocer dicha percepción es importante si se toma en cuenta que los alumnos suelen pasar más tiempo en las escuelas que en sus casas y que la educación es concebida como un vehículo para la movilidad social (Huerta, 2012; López-Calva et al., 2010). Por otra parte, conocer las percepciones de los apoderados al considerarse que la familia es fundamental en el desarrollo escolar de los estudiantes y que su apoyo tiene un efecto directo sobre los resultados académicos de estos (Ferry et al., 2000). Según los estudios de Kenny et al. (2003), realizados con estudiantes chinos cuyas familias migraron a Estados Unidos, los estudiantes demuestran un mejor desempeño escolar en lo académico y en las relaciones sociales con sus pares y profesores, cuando los padres apoyan su proceso educacional. En otro estudio realizado con migrantes mexicanos en Estados Unidos, se observó que el apoyo parental estaba estrechamente relacionado con el éxito en la escuela y con la intención de continuar en la educación superior (McWhirter et al., 1998). La familia, por lo tanto, tiene la capacidad de promover el desempeño educacional en sus hijos a través del fomento de las aspiraciones educacionales, el monitoreo de sus comportamientos sociales y el desarrollo de una relación fuerte entre padres e hijos (Perreira et al., 2006).

En comunidades migrantes, los estudiantes se ven obligados, en muchos casos sin quererlo, a ajustar sus vidas a un nuevo ambiente y a una nueva cultura. En estas circunstancias el soporte familiar puede traer consigo una mejor sensación de acogida en la localidad receptora (Kibria, 1994; White y Glick, 2000). Las investigaciones realizadas con adolescentes latinos que migraron a Estados Unidos han mostrado que, para ellos, los padres, hermanos y otros familiares son considerados como una fuente de apoyo fundamental en sus procesos de adaptación (Levitt, Guacci-Franco y Levitt, 1993; Martínez et al., 2004).

Junto con la familia, se ha demostrado que el apoyo de la escuela al estudiante migrante es muy importante y que una relación estrecha entre apoderados y profesores puede promover el éxito académico en los alumnos (Bankston y Zhou, 2002). Lo anterior se hace imprescindible entre familias migrantes que no comparten el mismo idioma de la comunidad de acogida, donde los padres perciben que sus hijos se benefician socialmente al hablar la lengua meta (L2) y donde la responsabilidad y preocupación de los profesores son percibidas como un tema clave para el éxito de sus hijos (Janssen et al., 2012). En efecto, el apoyo de los profesores es tan importante que puede ayudar a evitar el abandono escolar (Brewster y Bowen, 2004; García-Reid, Reid y Peterson, 2005; Martínez et al., 2004). Cuando los profesores crean una atmósfera de apoyo emocional esta puede conducir a una mayor posibilidad de aprendizaje en el estudiante y, por lo tanto, a un mayor interés en el proceso de aprendizaje (Rosenfeld et al., 2000). 
Los profesores, por su parte, generalmente no están preparados para educar a estudiantes de contextos culturales diferentes y menos aún a estudiantes que hablan una lengua distinta (Delgado-Gaitán, 1988). Según Herr y Anderson (2005), la mayoría de los profesores tienen problemas para percibir los valores culturales de estudiantes que no comparten su propio contexto cultural y esto puede llevar a malinterpretaciones; los profesores pueden interpretar como negativa una determinada actitud de un estudiante, a pesar de que en el contexto cultural propio de este su valoración sea positiva.

Como una vía para una educación multicultural en Chile, este trabajo propone un cuestionario cuyo objetivo es caracterizar las percepciones de los apoderados haitianos sobre los usos del creole, el francés y el español entre sus hijos, y las prácticas de alfabetización de estos en contextos escolares y familiares. Este instrumento también indaga sobre la motivación de los estudiantes en su aprendizaje (particularmente de ELE) y la relación que sus hijos construyen con la escuela, a fin de comprender los procesos de acogida que pueden favorecer el aprendizaje de la lengua meta. Este cuestionario está dirigido tanto a equipos directivos de los establecimientos como a los profesores de estos. Con este tipo de información, los agentes educacionales podrán repensar sus programas para considerar las percepciones de los apoderados, dado que estas son un factor considerable para el éxito escolar de los estudiantes migrantes.

De acuerdo con lo que hasta aquí hemos expuesto, nuestro objetivo general es diseñar y validar un cuestionario para caracterizar las percepciones de apoderados de estudiantes haitianos sobre prácticas de alfabetización y lenguas en contextos escolares (CPAHPAL). A partir de esto se analizarán la confiabilidad del cuestionario CPAHPAL mediante análisis psicométricos; la validez discriminante de las dimensiones uso de lenguas, motivación y relación con la escuela; y la validez del constructo a través de un Análisis Factorial Confirmatorio.

\section{MARCO TEÓRICO}

\subsection{LENGUA MATERNA (LM), SEGUNDA (L2) Y TERCERA LENGUA (L3)}

El concepto de lengua materna (LM) está estrechamente relacionado con el concepto de familia, especialmente los padres, pues son ellos los que la traspasan a través de las primeras relaciones sociales que tienen con sus hijos (King y Ennser-Kananen, 2013). La LM también se puede relacionar con la historia familiar, que incluye abuelos, tíos y otros familiares, en otras palabras, la LM no es solo un lexicón y una gramática particulares, sino una amplia conexión con la historia de la familia y la cultura, generación tras generación (Gregory, 2008). La familia migrante intenta crear oportunidades para mantener y usar su LM, en contextos escolares o domésticos, a la vez que intenta aprender la lengua meta del país de acogida (L2 y/o L3) (García et al., 2013; Wells, 2010). El esfuerzo de los padres por adquirir una L2 o L3 y mantener su LM puede estar relacionado con la percepción que tienen estos sobre la relevancia social del bilingüismo y de la educación bilingüe, además de su impacto sobre los logros académicos y económicos, pero, primordialmente, el deseo de mantener la LM se relaciona con la preservación de las raíces culturales (Stolzenberg y Tienda,1997; Tse, 2001). Por lo anterior, en el uso y mantención de la LM existe una relación afectiva que está relacionada con la construcción y la afirmación de la identidad (Kramsch, 2014; Pavlenko, 2007). 
El apoyo familiar para el uso de la LM, o la adquisición de la lengua de herencia, muestra un signo de unión entre la familia y el contexto social donde esta lengua puede ser usada, lo cual ayuda a enriquecer el dominio de la lengua materna y la propia cultura tras esa lengua. La familia es fundamental cuando la lengua propia es hablada por una minoría e intenta sobrevivir en un entorno donde existe otra lengua dominante, como es el caso de Chile en relación con el creole (García y Kleifgen, 2010; García et al., 2013). De acuerdo con las investigaciones de Castellotti y Moore (2002) y Tse (2001), es importante la percepción que los padres tienen acerca de la LM y de la L2/L3, porque cuando un padre siente que su idioma está en un nivel menor respecto a la lengua de la comunidad meta, se sentirá menos interesado en que sus hijos lo mantengan (Cooke y Simpson, 2012). Al respecto, Carreira y Kagan (2011) sostienen que en el fomento de las prácticas de alfabetización es fundamental que los alumnos mantengan su LM y, con ello, todo el bagaje cultural que esta conlleva. En su investigación un 71,3\% de los encuestados señalaron que se habían beneficiado de leer en su LM en casa, con su familia, pues esto les permitía sentirse parte de la comunidad y del grupo étnico familiares. Con esta actividad ellos podían comprender los códigos culturales comunes de su grupo, lo cual finalmente los ayudaba a sentirse orgullosos de sí mismos. Campano (2007) destaca la importancia del espacio informal (fuera de la sala de clases), donde el estudiante aprende a través de las experiencias e historias compartidas con otras personas. En una misma línea, Pahl y Kelly (2005) destacan que la alfabetización familiar es otro espacio clave para el aprendizaje, a través de interacciones que fomentan el compartir experiencias y formas de ver el mundo (Maguire y Graves, 2001).

Desde el punto de vista de la teoría sociocultural, aprender en diferentes contextos y situaciones sociales crea una transformación de las ideas previas que teníamos sobre algo, mediante la vivencia de nuevas experiencias. Dentro de esta perspectiva, los roles del lenguaje y la herencia cultural son claves, porque entregan al estudiante una cosmovisión y una forma de ver el mundo que es compartida con otros hablantes de su LM (Wertsch, 1991). Desde la perspectiva sociocultural, los diferentes contextos sociales cuentan con variados expertos o guías desde los que los estudiantes pueden aprender en sus interacciones (Vygotsky, 1978). Ahora bien, es importante que esta interacción sea activa por parte del aprendiente, pues a través de una interacción más activa la dimensión interpersonal tendrá una influencia mayor en el aprendizaje (Stone, 1998).

Conocer la forma en que los alumnos aprenden fuera de la clase (ambiente informal), con quién aprenden (interacción), y con qué recursos, puede ayudar a los docentes y autoridades escolares a entender cómo las experiencias del estudiante pueden fortalecer el proceso educativo formal en la sala de clases a través de la L2 o L3, valorando siempre la lengua materna (Jessel et al., 2011, Gregory, 2008).

\subsection{LA MOTIVACIÓN}

Según Gardner (1979), la motivación hacia la lengua meta está directamente relacionada con la identificación del aprendiente con el idioma, la cultura y los hablantes de la comunidad de esa lengua. Esta identificación con el idioma es un factor decisivo en la formación de la actitud y motivación hacia la L2 o L3. Esta actitud también se puede traducir en un intento por integrarse a la nueva comunidad por parte del hablante, lo que incluye no solo un interés en el estilo de vida y cultura de la comunidad meta, sino que una 
apertura general y un respeto hacia los otros grupos culturales (Gardner, 2001). De acuerdo con Ardasheva et al., (2012), la motivación hacia el aprendizaje de una lengua es el deseo de aprender ese idioma y se asocia con el esfuerzo y la actitud positiva hacia el estudio de una lengua determinada. La motivación es tomada en cuenta como un factor central para comprender el ritmo de aprendizaje de un idioma y el grado de competencia que puede alcanzar un aprendiz en la lengua meta (Ghazvini y Khajehpour, 2011; Oroujlou y Vahedi, 2011).

Frecuentemente, los antecedentes educacionales y socioculturales de los padres se vinculan con la actitud de los niños sobre el aprendizaje de una segunda lengua y también con el éxito en el aprendizaje de dicho idioma. Carhill et al. (2017) explican que la adquisición de una segunda o tercera lengua es un proceso que va más allá de la herencia de los padres. Otros factores que pueden afectar la motivación para aprender un idioma son: (a) factores personales (conocimientos previos, inteligencia, antecedentes personales, valores, personalidad, entre otros); o (b) factores situacionales (la estructura del curso, los programas de estudio, los métodos de enseñanza y de evaluación, las reglas de la clase y de la institución) (Dörnyei y Csizer, 2002; Ramburuth y McCormick, 2001). Las habilidades metacognitivas del estudiante, así como el apoyo docente, son aspectos que tienen un efecto positivo en la motivación (Brownlee et al., 2003; Mori et al., 2007), como también lo es el aprendizaje en un ambiente educacional democrático (Woolard y Gahng, 1990), donde no existe etnocentrismo y se respetan las culturas por igual.

Dado que la motivación es un factor decisivo para el aprendizaje de una lengua meta, y juega un papel primordial en el aprendizaje en contextos de inmigración, el cuestionario CPAHPAL considera la motivación de los estudiantes, según la percepción declarada por sus apoderados.

\subsection{RELACIÓN CON LA ESCUELA}

La percepción que los apoderados y los profesores tengan sobre el clima de la escuela tiene una influencia directa en el grado de deserción escolar, los problemas de disciplina, los problemas sicológicos y los resultados académicos (Payton et al., 2008; Rumberger, 1987; Wu et al., 1982). El clima escolar también está relacionado con la satisfacción laboral de los profesores y los esfuerzos de mejora escolar (Bryk et al., 2010; Miller et al.,1999). Grady et al. (2010) y Bukhari y Randall (2009), basados en la National Household Education Survey, indican que un $20 \%$ de los padres estadounidenses se mudaban desde sus barrios originales basados en el clima escolar, es decir, establecían sus viviendas de acuerdo con la escuela que mejor acogiera a sus hijos.

Los aprendientes de L2 o L3 en un país donde esta lengua es el idioma nativo (contexto de inmersión) tienen la oportunidad de exponerse directamente a situaciones de comunicación en la vida real. Las habilidades lingüísticas de estos aprendientes pueden ser desarrolladas más rápidamente que en un contexto formal escolar donde la L2/L3 se enseña como idioma extranjero, con pocas o nulas oportunidades de interacción con hablantes nativos. En Estados Unidos un 26\% de los estudiantes inmigrantes vive en situaciones en que sus familias están lingüísticamente aisladas y donde los padres tienen muy poca relación con las escuelas (Shields y Behrman, 2004). Por lo anterior, la relación que se cree entre los profesores, las autoridades de la escuela y la comunidad de padres es un nexo que debe fomentarse, pues ayudará a mejorar la percepción que los apoderados tengan acerca 
del establecimiento y ayudará a los estudiantes a sentirse acogidos por este nuevo ambiente de aprendizaje (Bryk et al., 2010).

Considerando lo que se ha expuesto, el cuestionario CPAHPAL incorporará la dimensión de relación de los apoderados haitianos con la escuela, para comprender en contexto la motivación y los logros académicos de sus hijos e hijas. Gracias al conocimiento de las percepciones de los apoderados, los colegios podrán tomar medidas, si así lo requieren, para mejorar las percepciones existentes.

\section{METODOLOGÍA}

\subsection{PARTICIPANTES}

La población de este estudio corresponde a apoderados haitianos de escuelas públicas de la Región Metropolitana. Para este estudio se decidió elegir una muestra no probabilística de 132 apoderados haitianos de 3 colegios municipales. Dos de los colegios están ubicados en la comuna de Estación Central y uno en la comuna de Quilicura. Los tres colegios poseen un grupo socioeconómico correspondiente al nivel medio-bajo. Se aplicó el cuestionario en estas comunas porque en ellas se distribuye el mayor porcentaje de haitianos a nivel nacional (Departamento de Extranjería y Migración, 2017). La distribución de los informantes según género fue de 62 hombres (47\%) y 70 mujeres (53\%). Las edades de quienes participaron en nuestro estudio fluctuaban entre los 29 y 38 años. Un $61 \%$ de los participantes llevaba entre uno y cuatro años en Chile. En cuanto al idioma, el creole era la lengua materna de todos y como segunda lengua, un $43 \%$ sabía francés y un $23 \%$ español. Un $66 \%$ no tenía escolaridad completa (menos de 12 años de escolaridad formal). Un 64\% aprendió ELE en Chile, un 14\% en República Dominicana y un 5\% en Haití. Es importante mencionar que para determinar el nivel de español nos basamos en la declaración de los participantes (dato auto reportado) y el resultado fue que aproximadamente la mitad (49\%) considera que tiene un nivel intermedio de este idioma. Dado el elevado número de participantes (132) era muy complejo tomarles una prueba formal de nivelación, que habría tardado dos horas en ser cumplimentada.

\subsection{CUESTIONARIO CPAHPAL}

El cuestionario CPAHPAL es un cuestionario de lápiz y papel con un tiempo aproximado de aplicación de 20-25 minutos. El propósito de este instrumento y sus instrucciones se explican en español y en creole, solicitando la firma del consentimiento informado a cada uno de los participantes en los mismos dos idiomas. El CPAHPAL cuenta con una sección en la que se solicitan datos personales y demográficos del apoderado con el fin de caracterizar la muestra, pero manteniendo el anonimato de los informantes. Los 54 ítems que componen el cuestionario se presentan organizados en las tres dimensiones que configuran el instrumento: uso de lenguas, motivación y relación con la escuela.

\subsubsection{Diseño y desarrollo del cuestionario CPAHPAL}

El cuestionario se construyó a partir de una extensa revisión bibliográfica y adaptando preguntas formuladas en el cuestionario de Gregory (2008) respecto al uso de las lenguas 
(LM y L2). El constructo que mide CPAHPAL está formado por las siguientes tres dimensiones que se explican a continuación.

\section{Dimensión 1: Lenguas}

Esta dimensión está conformada por tres secciones en las cuales se hacen las mismas preguntas considerando las tres lenguas, como se aprecia en la Tabla 1: (1) español, para conocer cómo el apoderado percibe el uso que el alumno hace de la lengua meta; (2) creole para conocer la percepción que tiene el apoderado sobre la lengua materna y si acaso su hijo la mantiene o intenta mantenerla; y (3) francés, para conocer el uso de esta en el contexto chileno. En esta dimensión se les pregunta a los apoderados sobre sus percepciones en cuanto al uso de la lengua por parte de su hijo (ej. su hijo habla en español con los profesores o su hijo lee libros en español). Dentro de esta dimensión se miden las habilidades o usos de la lengua (hablar, leer, escribir y escuchar), la interacción con otros a través de la lengua (familia, profesores y compañeros) y los recursos utilizados (escritos o audiovisuales) Gregory (2008).

Tabla 1. Número de preguntas de la primera dimensión

\begin{tabular}{|c|c|c|}
\hline Lenguas & \# preguntas & Número de pregunta \\
\hline Español & 8 & $1-8$ \\
\hline Creole & 8 & $9-16$ \\
\hline Francés & 8 & $17-24$ \\
\hline
\end{tabular}

\section{Dimensión 2: Motivación}

Con base en los factores definidos por Dörnyei y Csizer (2002) y Ramburuth y McCormick (2001), se crearon los ítems que muestra la Tabla 2 y que permiten captar: (1) la motivación hacia la escuela, es decir, la motivación hacia las actividades y personas que se encuentran en este contexto, tanto en clases como en recreos y actividades extracurriculares (ej. $\mathrm{Mi}$ hijo participa en actividades de la escuela); y (2) la motivación hacia el español, es decir, la motivación que tiene el alumno hacia el aprendizaje del idioma tanto en la escuela como en la casa. Asimismo, se busca caracterizar la relación del alumno con la interacción, hablada o escrita (ej. A mi hijo le gusta estudiar español en la casa).

Tabla 2. Número de preguntas de la segunda dimensión

\begin{tabular}{|c|c|c|}
\hline Motivación & \# preguntas & Número de pregunta \\
\hline Hacia la escuela & 14 & $25-38$ \\
\hline Hacia el español & 7 & $39-45$ \\
\hline
\end{tabular}




\section{Dimensión 3: Relación con la escuela}

En esta dimensión se describe la percepción que los apoderados tienen sobre las acciones de la escuela hacia ellos mismos (ej. la escuela me acoge) y hacia sus hijos; en particular, la forma en que la escuela enfrenta el desafío de tener alumnos migrantes, (ej. mi hijo me dice que no se siente discriminado en la escuela). La Tabla 3 muestra el número de preguntas relacionadas con esta dimensión.

Tabla 3. Número de preguntas de la tercera dimensión

\begin{tabular}{|c|c|c|}
\hline Dimensión & \# preguntas & Número de pregunta \\
\hline Relación con la escuela & 9 & $46-54$ \\
\hline
\end{tabular}

A diferencia de Gregory (2008), los ítems de CPAHPAL no son preguntas abiertas, sino alternativas tipo Likert. De esta forma, cada ítem quedó conformado por una aseveración y una escala tipo Likert de 5 opciones: siempre (puntuación 5), a veces (puntuación 4), rara vez (puntuación 3 ) y nunca (puntuación 2). Además, se incorporó la opción No aplica (puntuación 1), pensada para los ítems sobre lengua francesa, ya que no todos los apoderados la manejan. Otras razones que justifican la elección de la escala tipo Likert de 5 opciones fueron: la simplificación del posterior análisis, pues el empleo de esta escala permite una dicotomización de las respuestas y, en términos de aplicación, favorece la discriminación de los encuestados (Burnett, 2009). La escala tipo Likert también facilita la comprensión de los informantes y reduce el tiempo necesario para responder las preguntas (Black y Champion, 1976 y Saris y Gallhofer, 2007). Lo anterior es muy importante, si tomamos en cuenta que tuvimos una tasa importante de retorno de cuestionarios sin completar, por falta de tiempo de los informantes.

\subsubsection{Validación de contenido}

Una vez construido el instrumento se realizó la validación de contenido de este, para lo cual se les solicitó a tres expertos que revisaran los ítems del cuestionario (en español): un lingüista experto en ELE y dos psicólogos expertos en psicometría y construcción de encuestas. A los tres se les solicitó clasificar los ítems en tres grupos y puntuarlos de la siguiente manera: (3) No es necesario cambiar, (2) Podría necesitar un cambio y (1) Cambiar definitivamente. Una vez obtenidas las puntuaciones de los expertos se procedió a definir los cambios. Ninguno de los ítems obtuvo una puntuación de (1) y 8 de los ítems obtuvieron una puntuación (2), por lo que se decidió hacer algunos cambios. El instrumento fue creado en español y validado por expertos en esa lengua, sin embargo, debido a que sería completado por los apoderados de los alumnos, cuyo nivel de español no era requisito para la validación del cuestionario, se hizo una traducción del instrumento desde el español hacia el creole.

El pilotaje del cuestionario se realizó entre agosto y noviembre del año 2017 y contabilizó un total de 600 encuestas entregadas en las comunas de Estación Central (2 
colegios) y Quilicura (1 colegio). De estas 600 encuestas, el retorno fue de 132 (lo que equivale a un 22\%). Los cuestionarios fueron entregados a los equipos directivos quienes los enviaban a través de los estudiantes a sus casas.

Como el objetivo de este proyecto es la validación del cuestionario CPAHPAL, se realizó primero un estudio de validación considerando los 54 ítems inicialmente construidos (cuestionario estudio piloto). Luego, a partir de la información concluida del pilotaje y los análisis psicométricos realizados, se decidió eliminar 9 ítems y se volvieron a realizar los análisis para obtener un cuestionario confiable y validado de 45 ítems (cuestionario validado). Es importante mencionar que ambos análisis se realizaron con la misma muestra.

Con el objetivo de obtener los resultados de validación en ambos procedimientos, se realizó un análisis de confiabilidad a través del cálculo del índice de Alfa de Cronbach por dimensión. Posteriormente, para observar la correlación existente entre las dimensiones teóricas del instrumento, se realizó un análisis de correlación de Pearson, y así analizar la validez discriminante. Finalmente se llevó a cabo un análisis factorial confirmatorio (AFC) mediante extracción por factorización de ejes principales con rotación Promax, debido a que este instrumento está compuesto por tres dimensiones. Todos los análisis de esta investigación se realizaron con el Software IBM SPSS Statistics versión 22.

\subsubsection{Decisiones a partir del pilotaje}

Las tres dimensiones del instrumento se mantuvieron, pues se observó que presentaban una alta confiabilidad y, por lo tanto, una alta consistencia interna. Además, se confirmó la existencia de tres factores a través del método AFC, según el cual los constituyentes agrupaban casi los mismos reactivos que la versión teórica del instrumento.

En el análisis por dimensión se puede mencionar que: la dimensión 1 (Uso de lenguas), que corresponde a los ítems relacionados con el uso del español, francés y creole, se mantuvo igual, con 24 ítems subdivididos en los tres grupos.

En cuanto a la dimensión 3 (Relación con la escuela), que tiene ítems relacionados con la percepción del apoderado hacia la escuela, se mantuvieron los mismos ítems de la dimensión original, sin embargo, se decidió agregar dos ítems de la subdimensión Motivación hacia la escuela (que pertenecía a la dimensión 2), pues estaban más relacionados con esta área y, según los resultados estadísticos, también poseía una correlación mayor con esta.

Finalmente, en la dimensión 2 correspondiente a la Motivación y que originalmente estaba subdividida entre motivación hacia la escuela y hacia el español, se presentó la mayor cantidad de eliminación de ítems por problemas en cuanto al peso factorial de estos. En el resultado del AFC, se observó que varios ítems no cargaban en ninguno de los tres factores principales. Además, varios de esos mismos ítems restaban confiabilidad a la dimensión.

\section{RESULTADOS DEL CUESTIONARIO VALIDADO}

A continuación, se presentan los resultados del estudio de validación del cuestionario CPAHPAL, conformado por los 45 ítems que se escogieron después de los análisis del instrumento piloto. En la Tabla 4 se presenta la distribución de preguntas por dimensiones y subdimensiones. Es importante mencionar que esta validación se realizó usando la misma muestra del pilotaje. 
Tabla 4. Dimensiones del cuestionario validado

\begin{tabular}{|l|l|c|c|}
\hline \multicolumn{1}{|c|}{ Dimensión } & Subdimensión & \# Preguntas & Número de ítem \\
\hline Dimensión 1: & Español & 8 & $1-8$ \\
\hline Lenguas & Creole & 8 & $9-16$ \\
\hline & Francés & 8 & $17-24$ \\
\hline $\begin{array}{l}\text { Dimensión 2 } \\
\text { Motivación }\end{array}$ & -- & 10 & $25-34$ \\
\hline $\begin{array}{l}\text { Dimensión 3 } \\
\text { Relación con la escuela }\end{array}$ & -- & 11 & $35-45$ \\
\hline
\end{tabular}

\subsection{CONFIABILIDAD DEL CUESTIONARIO CPAHPAL}

Para determinar la confiabilidad del cuestionario CPAHPAL, se efectuó una medición mediante el análisis de Alfa de Cronbach, cuyos resultados se muestran en la Tabla 5.

Tabla 5. Valores de confiabilidad según Alfa de Cronbach

\begin{tabular}{|c|c|l|}
\hline Alfa de Cronbach & N de elementos & \multicolumn{1}{|c|}{ Dimensión } \\
\hline .89 & 45 & Cuestionario completo \\
\hline .88 & 24 & Dimensión 1 Lenguas \\
\hline .76 & 10 & Dimensión 2 Motivación hacia español \\
\hline .92 & 11 & Dimensión 3 Relación con la escuela \\
\hline
\end{tabular}

Luego de revisar los valores del Alfa de Cronbach, tanto del cuestionario completo como de cada una de las dimensiones, podemos confirmar que la confiabilidad del instrumento es muy alta. Esto quiere decir que, si se realiza nuevamente el test, las respuestas tenderían a repetirse en todas las dimensiones, incluso en la de motivación, pues este resultado, a pesar de ser el menor, es aceptable para una investigación exploratoria como esta (George y Mallery, 2003; Gliem y Gliem, 2003).

\subsection{VALIDEZ DISCRIMINANTE DEL CUESTIONARIO CPAHPAL}

Para determinar la validez discriminante del cuestionario CPAHPAL, primero se realizó un análisis de Correlación de Pearson con el objeto de verificar las correlaciones entre las tres dimensiones teóricas conformadas por los ítems definitivos. El resultado del análisis anterior se muestra en la Tabla 6. 
Tabla 6. Correlación de Pearson entre las dimensiones

\begin{tabular}{|l|c|c|c|}
\hline & $\mathbf{1}$ & $\mathbf{2}$ & $\mathbf{3}$ \\
\hline 1. Lenguas & --- & $.31 *$ & $.04 *$ \\
\hline 2. Motivación & $.31 *$ & --- & $.34 *$ \\
\hline 3 Relación con la escuela & $.04 *$ & $.34 *$ & --- \\
\hline
\end{tabular}

Nota. * valor $\mathrm{p}<.005$

A partir del resultado se observa que existe una correlación baja positiva entre la dimensión 1 Uso de lenguas y la dimensión 2 Motivación. El mismo patrón se observa para la relación entre la dimensión 2 Motivación y la dimensión 3 Relación con la escuela. Una correlación positiva muy baja, en cambio, se observa entre Uso de lenguas y Relación con la escuela. Este resultado es consistente con la definición de cada dimensión, ya que la motivación está relacionada con el uso de las lenguas y la relación con la escuela, pero esta última no está directamente relacionada con el uso de las lenguas. Además, podemos observar que disminuyó bastante la correlación existente entre Uso de lenguas y Motivación, respecto a los resultados del piloto, lo cual quiere decir que la eliminación de los ítems seleccionados permitió diferenciar claramente entre dimensiones; en otras palabras, las dimensiones miden constructos diferentes.

Luego de revisar la correlación entre las dimensiones (Tablas 7 y 8), procedimos a observar cómo se comportaban los ítems luego de aplicar un AFC de tres factores, con el objetivo de analizar la validez de constructo.

Tabla 7. Varianza total explicada

\begin{tabular}{|c|c|c|c|}
\hline & Total & \% de varianza & $\%$ acumulado \\
\hline 1 & 10,69 & 23,75 & 23.75 \\
\hline 2 & 6,64 & 14,75 & 38.49 \\
\hline 3 & 5,01 & 11,14 & 49.63 \\
\hline
\end{tabular}

Tabla 8. Matriz de componente

\begin{tabular}{|c|c|c|c|}
\hline \multirow{2}{*}{} & \multicolumn{3}{|c|}{ Componente } \\
\cline { 2 - 4 } & 1 & 2 & 3 \\
\hline P1 & .395 & & \\
\hline P2 & .265 & & \\
\hline P3 & .461 & & \\
\hline P4 & .246 & & \\
\hline
\end{tabular}


Estudios Pedagógicos XLV, $\mathrm{N}^{\circ}$ 3: 51-77, 2019

CUESTIONARIO DE APODERADOS DE ESTUDIANTES HAITIANOS SOBRE PRÁCTICAS DE ALFABETIZACIÓN Y LENGUAS

\begin{tabular}{|c|c|c|c|}
\hline P5 & .419 & & \\
\hline P6 & .204 & & \\
\hline P7 & .337 & & \\
\hline P8 & .500 & & \\
\hline P9 & .385 & & \\
\hline P10 & .479 & & \\
\hline P11 & .551 & & \\
\hline P12 & .591 & & \\
\hline P13 & .626 & & \\
\hline P14 & .732 & & \\
\hline P15 & .399 & & \\
\hline P16 & .592 & & \\
\hline P17 & .795 & & \\
\hline P18 & .807 & & \\
\hline P19 & .814 & & \\
\hline P20 & .799 & & \\
\hline P21 & .835 & & \\
\hline P22 & .622 & & \\
\hline P23 & .827 & & \\
\hline P24 & .823 & & \\
\hline P28 & & & .437 \\
\hline P29 & & & .531 \\
\hline P33 & & & .425 \\
\hline P34 & & & .511 \\
\hline P36 & & & .469 \\
\hline P37 & & & .563 \\
\hline P38 & & & .489 \\
\hline P39 & & & .506 \\
\hline P40 & & & .504 \\
\hline P42 & & & .258 \\
\hline P43 & & & .464 \\
\hline P45 & & & .505 \\
\hline P46 & & .717 & \\
\hline P47 & & .683 & \\
\hline
\end{tabular}




\begin{tabular}{|l|l|l|l|}
\hline P48 & & .727 & \\
\hline P49 & & .717 & \\
\hline P50 & & .633 & \\
\hline P51 & & .624 & \\
\hline P52 & & .772 & \\
\hline P53 & & .767 & \\
\hline P54 & & .738 & \\
\hline
\end{tabular}

En las Tablas 7 y 8, se observa que existen tres dimensiones que configuran el constructo que mide el cuestionario CPAHPAL. La varianza explicada apoya la idea de mantener los tres factores teóricos, aunque hay algunos ítems: 1, 2, 4, 5, 6, 7, 8 (Ver anexo 1) que no cargan solo en un factor. Es interesante observar que esos ítems referidos al uso del español también cargan en la dimensión de motivación hacia la lengua, sin embargo, se decidió dejar los primero ocho ítems en la primera dimensión, pues están muy relacionados con las otras preguntas sobre el creole y el francés.

\section{CONCLUSIONES}

En esta investigación, se ha diseñado y validado, para un uso específico, un instrumento para medir las percepciones de los apoderados haitianos acerca de las prácticas de alfabetización y lenguas en contextos escolares: CPAHPAL.

El estudio piloto permitió mejorar el instrumento ya que en la versión inicial las dimensiones de motivación y uso de lenguas presentaban una correlación muy alta. Lo anterior se ve bastante respaldado por las investigaciones de Gardner (1979) para quien la motivación hacia una lengua y el uso de la lengua están muy correlacionados. El estudio piloto y el análisis a nivel de dimensión permitieron detectar cuáles eran los ítems que cargaban en ambas dimensiones o que restaban confiabilidad. De este modo, el cuestionario definitivo cuenta con ítems para caracterizar dimensiones que miden factores distintos entre ellos, lo que se ve corroborado por la correlación baja entre dimensiones y por el análisis factorial confirmatorio. El cuestionario definitivo cuenta con una dimensión de motivación orientada hacia el español y permite conocer de mejor manera qué temas son los que motivan al estudiante a aprender una lengua y cuáles están más bien vinculados con la asistencia a la escuela.

El análisis factorial confirmatorio muestra que el constructo que considera tres dimensiones para la caracterización de las percepciones de los apoderados sobre las lenguas y sus contextos de uso es adecuado. Por lo tanto, es importante no solo conocer las percepciones de los apoderados acerca de las prácticas de alfabetización y de uso del español sino también del creole, pues se busca legitimar el uso de la lengua materna en espacios de interacción familiar. El cuestionario no solo se focaliza en el uso de la LM y de las L2 y L3 en la escuela, sino también en cómo los alumnos aprenden fuera de la clase (ambiente informal). De igual forma, considera con quiénes aprenden (interacción) y con 
qué recursos (Jessel et al., 2011; Gregory, 2008). Junto con las prácticas de alfabetización y uso de las lenguas, considera factores clave motivacionales y contextuales que pueden favorecer el aprendizaje del español en contexto de migración.

Tras los análisis estadísticos de validación podemos afirmar que este instrumento logró una confiabilidad óptima $(\alpha=.89)$. Asimismo, mediante un análisis factorial confirmatorio se determinó la existencia de las tres dimensiones que conforman el constructo medido. De esta manera se logró validar un cuestionario en creole conformado por 45 ítems, que puede ser utilizado por los establecimientos educativos para levantar las percepciones de los apoderados acerca de las prácticas de alfabetización y los usos de las lenguas en comunidades migrantes. El cuestionario CPAHPAL caracteriza las percepciones de los apoderados haitianos sobre (1) los usos y prácticas de alfabetización y de lenguas, considerando tanto la lengua materna como las L2 y L3; (2) la motivación hacia el español; y (3) la relación con la escuela. Hasta ahora se contaba solo con preguntas abiertas para suscitar una conversación con los padres (Gregory, 2008), pero no se disponía de un cuestionario válido, confiable y de fácil cumplimentación para este proceso. Las preguntas de la dimensión lenguas se basaron en las investigaciones de Gregory (2008); sin embargo, consideramos que el hecho de contar con un cuestionario con respuesta cerrada permitirá levantar en menor tiempo y a mayor escala las percepciones de los apoderados haitianos.

Otro aspecto destacable del cuestionario es que está escrito en creole; contar con un cuestionario en creole es fundamental para un grupo migrante que es minoría en Chile, pues facilita el proceso de acogida de dicho grupo (García y Kleifgen, 2010; García et al., 2013).

A través de este cuestionario, los establecimientos educacionales podrán tener la posibilidad de conocer las percepciones de los grupos migrantes haitianos, y así podrán dar una mejor acogida a este grupo. Tal como destaca la investigación en el área, es importante contar con instrumentos que levanten y validen la percepción de los apoderados para que sientan que el colegio les abre las puertas y, de esta forma, la acogida se traduzca en que todos los agentes de la comunidad escolar se sientan parte del proceso educativo (Kenny et al., 2003; Ferry et al., 2000).

El objetivo es que, con esta información, el profesor (o directivo) pueda conocer la percepción de los apoderados para, desde este conocimiento, tomar decisiones para mejorar los procesos de acogida. A partir de esta información más general, los equipos directivos podrían reunirse con los apoderados para informarles sobre lo que se está haciendo en clases o podrían conversar con los apoderados sobre la importancia de mantener la lengua materna en los estudiantes, pues no solo sirve para comunicarse con la familia, sino también para mantener y valorar la carga identitaria de la persona.

Si bien una limitación de este estudio fue la baja tasa de retorno de los instrumentos cumplimentados, es importante mencionar que en las escuelas donde los directores y los profesores estaban más comprometidos con los apoderados haitianos (se comunicaban con ellos cada semana, les preguntaban sobre sus necesidades y dudas sobre el colegio) la tasa de respuesta fue muy alta, lo que demuestra que cuando el apoderado se siente parte fundamental del proceso educativo de sus hijos, ellos responden de una mejor manera y de esa forma participan de este tipo de instancias (Bryk et al., 2010). Lo anterior también se demostraba en el compromiso de los estudiantes por entregar las encuestas a tiempo.

Por último, es importante mencionar que el presente cuestionario fue validado con la misma muestra con la que se hizo el pilotaje, por lo tanto, sus resultados deben ser 
tomados en cuenta con cierta cautela. En efecto, sería recomendable realizar entrevistas a los apoderados para poder sacar mejores conclusiones de sus percepciones.

\section{REFERENCIAS BIBLIOGRÁFICAS}

Ardasheva, Y., Tong S. \& Tretter, T. (2012). Validating the English language learners motivation scales: pre-college to measure language learning motivational orientation among young ELLS. Learning and individual differences, 22, 473-483.

Bankston, C. L. \& Zhou, M. (2002). Social capital and immigrant children's achievement. Schooling and Social Capital in Diverse Cultures, 13, 13-39.

Black, J. A. and Champion D. J. (1976). Method and issues in social research. Nueva York: John Wiley \& Sons.

Brewster, A. B. \& Bowen, G. L. (2004). Teacher support and the school engagement of Latino middle and high school students at risk of school failure. Child and Adolescent Social Work Journal, 21(1), 47-67. doi: 10.1023/B:CASW.0000012348.83939.6b.

Brownlee, J., Purdie, N. y Boulton-Lewis, G. (2003). Paradigm shifts in pre-service teacher education students: Case studies of chang- es in epistemological beliefs. Australian Journal of Educational and Developmental Psychology, 3(1), 1-6.

Bryk, A. S., Sebring, P. B., Allensworth, E., Luppescu, S. \& Easton, J. (2010). Newbook by Consortium Researchers Identifies Proven formula for Successful School Reform Chicago. Recuperado de https://consortium.uchicago.edu/sites/default/files/publications/OrganizingSchoolPressRelease.pdf.

Bukhari, P., Randall, E. V. (2009). Exit and entry: why parents in Utah left public schools and chose private schools. Journal of School Choice, 3(3): 242-270.

Burnett, J. (2009). Doing your social science dissertation, London: SAGE Publications.

Campano, G. (2007). Immigrant students and literacy: Reading, writing and remembering. New York: Teachers College Press.

Carhill, A., Suárez-Orozco, C. \& Páez, M. (2017). Explaining English language proficiency among adolescent immigrant students. American Educational Research Journal, 45, 1155 - 1179.

Carreira, M. \& Kagan, O. (2011). The results of the national heritage language survey: implications for teaching, curriculum design and professional development. Foreign Language Annals, 44, 40-64. doi:10.1111/j.1944- 9720.2010.01118.

Castellotti, V. \& Moore, D. (2002). Social representations of languages and teaching. Guide for the development of language education policies in Europe from linguistic diversity to plurilingual education. Reference study for the council of Europe. Recuperado de: https://www.coe.int/t/dg4/ linguistic/source/castellottimooreen.pdf.

Cooke M. \& Simpson J. (2012). Discourses about linguistic diversity. En The Routledge Handbook of Multilingualism. In Martin-Jones M, Blackledge A. \& A. Creese (Eds.), Routledge Handbooks in Applied Linguistics, (pp. 116-130). London: Routledge.

Delgado-Gaitan, C. (1988). The value of conformity: learning to stay in school. Anthropology and Education. 4(19), 354-381, doi: https://doi.org/10.1525/aeq.1988.19.4.05x09221.

Departamento de Extranjería y Migración. (2018). Población migrante en Chile. 3 marzo de 2018, Ministerio del Interior, Chile Sitio web: http://www.extranjeria.gob.cl/media/2017/09/RM_ PoblacionMigranteChile 1.pdf.

Dörnyei, Z. \& Csizér, K. (2002). Some dynamics of language attitudes and motivation: Results of a longitudinal nationwide survey. Applied Linguistics, 23, 421-462.

Ferry, T. R., Fouad, N. y Smith, P. L. (2000). The role of family context in a social cognitive model for career-related choice behavior: a math and science perspective. Journal of Vocational Behavior, 57(3), 348-364, doi: 10.1006/jvbe.1999.1743. 
García, O. \& Kleifgen, J. (2010). Educating English language learners as emergent bilinguals: Policies, programs and practices for English language learners. New York: Teachers College Press.

García, O., Zakharia, Z. \& Otcu, B. (2013). Bilingual Community Education for American Children: Beyond Heritage Languages in a Global City. Bristol, UK: Multilingual Matters.

Garcia-Reid, P., Reid, R. J. \& Peterson, N. A. (2005). School engagement among Latino youth in an urban middle school context: Valuing the role of social support. Education and Urban Society, 47(3), 328-343.

Gardner, R. C. (1979) Social psychological aspects of second language acquisition. In H. Giles and R. St. Clair (Eds), Language and Social Psychology (pp. 193-220). Oxford: Basil Blackwell. . (2001). Integrative motivation: past, present, and future. Distinguished Lecture Series, Temple University Japan. Recuperado de http://publish.uwo.ca/ gardner/docs/GardnerPublicLecture1. pdf.

George, D. \& Mallery, P. (2003). SPSS for Windows step by step: A simple guide and reference. Boston: Allyn \& Bacon.

Ghazvini, S. D. \& Khajehpour M. (2011). Attitudes and motivation in learning English as second language in high school students. Procedia Social and Behavioral Sciences, 15, 1209-1213.

Gliem, J. A. \& Gliem, R. R. (2003). Calculating, interpreting, and reporting cronbach's alpha reliability coefficient for likert-type scales. Presentada en Midwest Research to Practice Conference in Adult Continuing and Community Education. Ohio State University, Ohio.

Grady, S., S. Bielick \& S. Aud (2010). Trends in the Use of School Choice: 1993 to 2007. Statistical Analysis Report. Recuperada de https://nces.ed.gov/pubs2010/2010004.pdf.

Gregory, E. (2008). Learning to read in a new language. Los Angeles: Sage.

Herr, K. \& Anderson, G.L. (2005). The action research dissertation: A guide for students and faculty. Thousand Oaks: Sage Pub.

Huerta Wong, J. (2012). El rol de la educación en la movilidad social de México y Chile. ¿La desigualdad por otras vías? Revista Mexicana de Investigación Educativa, 17(52), 65-88.

Janssen, M., Bakker, J., Bosman, A., Rosenberg, K. \& Leseman, P. (2012). Differential trust between parents and teachers of children from low-income and immigrant backgrounds. Educational Studies, 38(4), 386-396.

Jessel, J., Kenner, C., Gregory, E., Ruby, M. \& Arju, T. (2011). Different spaces: Learning and literacy with children and their grandparents in east London homes. Linguistics and Education, 22, 37-50.

Kenny, M., D. Blustein, Chaves, A., Grossman, J. \& Gallagher, L. (2003). The role of perceived barriers and relational support in the educational and vocational lives of high school students. Journal of Counseling Psychology, 50(2). 142-155. doi: 10.1037/0022-0167.50.2.142.

Kibria, N. (1994). Household structure and family ideologies: the dynamics of immigrant economic adaptation among Vietnamese refugees. Social Problems, 41(1). doi: 41. 81-96. 10.1525/ sp.1994.41.1.03x0426j.

King, K. A. \& Ennser-Kananen, J. (2013). Heritage languages and language policy. In C. A. Chapelle (Ed.), Encyclopaedia of Applied Linguistics.

Kramsch, C. (2014). Teaching foreign languages in an era of globalization: Introduction. Modern Language Journal, 98, 296-311.

Levitt, M. J., Guacci-Franco, N. \& Levitt, J. L. (1993). Convoys of social support in childhood and early adolescence: Structure and function. Developmental Psychology, 29(5), 811-818. Recuperado de http://dx.doi.org/10.1037/0012-1649.29.5.811.

López-Calva, L. F. y Macías, A. (2010). “Estudias o trabajas?” Deserción escolar, trabajo temprano y movilidad en México. En Serrano, Julio y Florencia Torche (Eds.), Estudios de movilidad social en México (pp. 165-187), Ciudad de México: Centro de Estudios Espinosa Yglesias.

Maguire, M. H. \& Graves, B. (2001). Speaking Personalities in Primary School Children's L2 
Writing. TESOL Quarterly, 35(4), 561-593.

Martínez, C. R., De Garmo, D. S. \& Eddy, M. J. (2004). Promoting Academic Success Among Latino Youth. Hispanic Journal of Behavioral Sciences. 26(2). 128-151. doi: 10.1177/0739986304264573.

McWhirter, E. H., Hackett, G., y Bandalos, D. L. (1998). A causal model of the educational plans and career expectations of Mexican American high school girls. Revista Journal of Counseling Psychology, 45(2), 166-181. doi: http://dx.doi.org/10.1037/0022-0167.45.2.166.

Miller, M. D., Brownell, M. T. \& Smith, S. W. (1999). Factors that predict teachers staying in, leaving or transferring from the special education classroom. Exceptional Children, 65, 201-218.

Mori, Y., Sato, K. \& Shimizu, H. (2007). Japanese Language Students' Perceptions on Kanji Learning and Their Relationship to Novel Kanji Word Learning Ability. Language Learning, 57, 57-85. doi:10.1111/j.1467-9922.2007.00399.

Oroujlou, N. \& Vahedi, M. (2011). Motivation, attitude, and language learning. Procedia - Social and Behavioral Sciences, 29, 994-1000.

Pahl, K. \& Kelly, S. (2005). Family Literacy as a Third Space Between Home and School: Some Case Studies. Literacy, 39(2), 91-96.

Pavlenko, A. (2007). Autobiographic Narratives as Data in Applied Linguistics. Applied Linguistics, $28,163-188$

Pérez, C. (2004). Técnicas de Análisis Multivariante de Datos. Aplicaciones con SPSS. Madrid: Pearson Education.

Pérez, T. (2016). Fronteras imaginarias en América latina. La experiencia migratoria de haitianos en Chile. Revista Rumbos TS. Un espacio crítico para la reflexión en Ciencias Sociales, (3), 69-82. Recuperado de http://revistafacso.ucentral.cl/index.php/rumbos/article/view/23/19>.

Perreira, K. M., Harris, K. M. \& Lee, D. (2006). Making it in America: High school completion by immigrant and native youth. Demography, 43(3), 511- 536.

Payton, J., Weissberg, R. P., Durlak, J. A., Dymnicki, A. B., Taylor, R. D., Schellinger,

K. B. \& Pachan, M. (2008). The positive impact of social and emotional learning for kindergarten to eighth-grade students: Findings from three scientific reviews. Recuperado de https://www. casel.org/wp- content/uploads/2016/08/PDF-4-the-positive-impact-of-social-and- emotionallearning-for-kindergarten-to-eighth-grade-students-executive-summary.pdf.

Ramburuth, P. \& McCormick, J. (2001). Learning diversity in higher education: A comparative study of international Asian and Australian students. Higher Education, 42(3), 333-350.

Riedemann, A. y Stefoni, C. (2015). Sobre el racismo, su negación, y las consecuencias para una educación anti-racista en la enseñanza secundaria chilena. Revista Latinoamericana Polis, 42(14), 191-216. Recuperado de https://scielo.conicyt.cl/pdf/polis/v14n42/art_10.pdf.

Rosenfeld, L. B., Richman, J. M. \& Bowen, G. L. (2000). Social support networks and school outcomes: The centrality of the teacher. Child and Adolescent Social Work Journal, 17(3), 205226. doi: 10.1023/A:1007535930286.

Rumberger, R. (1987). High school dropouts: A review of issues and evidence. Review of Education Research, 57(2). doi:10.2307/1170232.

Saris, W. E. \& Gallhofer, I. N. (2007). Design, evaluation and analysis of questionnaires for survey research. Nueva York, Hoboken: John Wiley \& Sons.

Shields, M. K. \& Behrman, R. E. (2004). Children of immigrant families: Analysis and recommendations. The Future of Children, 14(2): 4-15. doi:10.2307/1602791.

Stefoni, C., Acosta, E., Gaymer, M., y Casas-Cordero, F. (2008). Niños y niñas inmigrantes en Santiago de Chile. Entre la integración y la exclusión. Recuperado de http://www.deustopublicaciones.es/ deusto/pdfs/cuadernosdcho/cuadernosdcho58.pdf.

Stolzenberg R. M. \& Tienda M. (1997). English proficiency, education, and the conditional economic assimilation of Hispanic and Asian origin men. Social Science Research, 26(1), 25-51.

Stone, C. B. (1998). Leveling the playing field: An urban school system examines access to 
mathematics curriculum. Urban Review, 30(4), 295-307.

Tse, L. (2001). Why don't they learn English? Separating fact from fallacy in the U.S. language debate. New York: Teachers College Press.

Valenzuela P., Riveros, K., Palomo, N., Araya, O., Campos, B. Salazar, C. y Tavie, C. (2014). Integración laboral de los inmigrantes haitianos, dominicanos y colombianos en Santiago de Chile. Revista Antropologías del Sur, (2), 101-121.

Vygotsky, L. S. (1978). Mind in Society. Cambridge, MA: Harvard University Press.

Wells, R. S. (2010). Children of immigrants and educational expectations: The roles of school composition. Teachers College Record, 112(6), 1679-1704.

Wertsch, J. V. (1991). Voces de la mente. Un enfoque sociocultural para el estudio de la acción mediada. Madrid: Visor.

White, M., \& Glick, J. (2000). Generation Status, Social Capital, and the Routes out of High School. Revista Sociological Forum, 15(4), 671-691. Recuperado de http://www.jstor.org/stable/684978.

Woolard, K. A. \& Gahng, T. (1990). Changing language policies and attitudes in autonomous Catalonia. Language in Society 19, 311-330.

Wu, S., Pink, W., Crain, R. \& Moles, O. (1982). Student suspension: A critical reappraisal. The Urban Review. 14(4), 245-303, doi: https://doi.org/10.1007/BF02171974. 


\section{ANEXO 1 Cuestionario validado}

\section{Cuestionario CPAHPAL en español \\ Estudio sobre percepción de prácticas de alfabetización y lenguaje Cuestionario padres o apoderados}

La finalidad de esta encuesta es conocer su percepción como padre(madre) o apoderado(a)

1. Sobre los idiomas, las actividades de lectura y escritura de la escuela y la casa.

2. La motivación de su hijo(a) por la escuela y el español.

3. La relación con la escuela.

La idea es poder compartir estas percepciones con los docentes y autoridades de las escuelas. Así se podrá incorporar su visión para llevar cambios que mejoren las oportunidades de aprendizaje ofrecidas a su hijo(a).

Le agradecemos responder este cuestionario del modo más claro posible. Las respuestas al cuestionario y los datos personales son confidenciales y solo serán utilizadas para fines de este estudio.

Para hacerlo le agradecemos seguir las siguientes instrucciones:

Por favor, conteste todas las preguntas de este cuestionario.

$>$ Lea atentamente cada pregunta y marque con una equis $(\mathrm{X})$ el casillero que corresponda a su respuesta. Siempre se debe marcar un solo casillero.

$>\quad$ Si se equivoca en su respuesta, rellene todo el casillero y marque una nueva equis (X) en el casillero correcto.

$>\quad$ Cuando se le pida anotar números y respuestas escritas, por favor hágalo de manera clara, precisa y fácil de leer.

$>\quad$ Una vez contestado el cuestionario entréguelo a la persona encargada en el colegio.

$>$ La segunda parte de esta encuesta consiste en una breve respuesta a dos preguntas.

¡Muchas gracias por su cooperación! 


\section{Datos generales}

\section{Datos personales}

1. Nombre

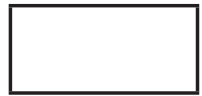

2. Apellido Paterno

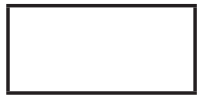

3. Apellido Materno

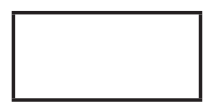

4. Género: Masculino Femenino

5. Edad:

6. Años en Chile:

7. Idioma materno:

8. Otros idiomas:

9. Nivel de español: (1) Bajo (2) Intermedio (3)Avanzado (4)Superior

10. Años de escolaridad completos:

11. ¿Dónde aprendió español?:

12. ¿Trabaja actualmente?: Sí

$$
\text { No }
$$

\section{Datos vivienda}

13. ¿En qué comuna vive actualmente?:

14. ¿Con cuántas personas vive actualmente?

15. ¿Cuántos hijos tiene?:

A continuación, por favor enliste los hijos que viven con usted:

\begin{tabular}{|c|c|c|c|c|c|c|}
\hline Nombre & $\begin{array}{l}\text { Apellido } \\
\text { paterno }\end{array}$ & $\begin{array}{l}\text { Apellido } \\
\text { materno }\end{array}$ & Sexo & Edad & $\begin{array}{l}\text { Nació } \\
\text { en Chile }\end{array}$ & $\begin{array}{l}\text { Curso } \\
\text { actual } \\
(2017)\end{array}$ \\
\hline & & & ${ }^{\mathrm{M}} \_\mathrm{F}$ & & $\begin{array}{r}\text { Sí } \\
\\
\end{array}$ & \\
\hline & & & $-^{\mathrm{M}}-^{\mathrm{F}}$ & & $\begin{array}{c}\text { Sí } \\
\\
\end{array}$ & \\
\hline & & & ${ }_{-\mathrm{M}} \_\mathrm{F}$ & & $\begin{array}{c}\text { Sí } \\
\\
\end{array}$ & \\
\hline & & & ${ }_{-\mathrm{M} \_\mathrm{F}}$ & & $\begin{array}{r}\text { Sí } \\
\\
\end{array}$ & \\
\hline & & & ${ }_{-}{ }_{-} \mathrm{F}$ & & $\begin{array}{l}\text { Sí } \\
\\
\end{array}$ & \\
\hline
\end{tabular}

\section{Primera parte}

Recuerde marcar con una equis $(\mathrm{X})$ la opción más adecuada según su percepción. Si considera que la afirmación no lo representa, elija No Aplica (N/A).

\section{Uso de los idiomas}




\section{Sobre español}

\begin{tabular}{|c|c|c|c|c|c|c|}
\hline & Indicador & Siempre & $A$ veces & Rara vez & Nunca & N/A \\
\hline 1 & $\begin{array}{l}\text { Mi hijo(a) habla español en la casa con los integrantes de la } \\
\text { familia. }\end{array}$ & & & & & \\
\hline 2 & $\begin{array}{l}\text { Mi hijo(a) lee (libros, revistas, cosas en internet, etc.) en } \\
\text { español en la casa. }\end{array}$ & & & & & \\
\hline 3 & $\begin{array}{l}\text { Mi hijo(a) escucha o ve música o videos (por internet o en la } \\
\text { radio, etc.) en español en la casa }\end{array}$ & & & & & \\
\hline 4 & Mi hijo(a) habla en español con los profesores en la escuela. & & & & & \\
\hline 5 & Mi hijo(a) habla en español con los compañeros en la escuela. & & & & & \\
\hline 6 & Mi hijo(a) lee (textos) en español en la escuela. & & & & & \\
\hline 7 & Mi hijo(a) escribe en español en la casa. & & & & & \\
\hline 8 & Mi hijo(a) escribe en español en la escuela. & & & & & \\
\hline
\end{tabular}

\section{Sobre creole}

\begin{tabular}{|c|c|c|c|c|c|c|}
\hline & Indicador & Siempre & A veces & Rara vez & Nunca & N/A \\
\hline 9 & $\begin{array}{l}\text { Mi hijo(a) habla creole en la casa con los integrantes de la } \\
\text { familia. }\end{array}$ & & & & & \\
\hline 10 & $\begin{array}{l}\text { Mi hijo(a) lee (libros, revistas, cosas en internet, etc.) en } \\
\text { creole en la casa. }\end{array}$ & & & & & \\
\hline 11 & $\begin{array}{l}\text { Mi hijo(a) escucha música o videos (por internet o en la radio, } \\
\text { etc.) en creole en la casa. }\end{array}$ & & & & & \\
\hline 12 & Mi hijo(a) habla en creole con los profesores en la escuela. & & & & & \\
\hline 13 & Mi hijo(a) habla en creole con los compañeros en la escuela. & & & & & \\
\hline 14 & Mi hijo(a) lee (textos) en creole en la escuela. & & & & & \\
\hline 15 & Mi hijo(a) escribe en creole en la casa. & & & & & \\
\hline 16 & Mi hijo(a) escribe en creole en la escuela. & & & & & \\
\hline
\end{tabular}

Sobre Francés (Si no habla francés, no complete estos ítems y continue con el item 25)

\begin{tabular}{|c|c|c|c|c|c|c|}
\hline & Indicador & Siempre & A veces & Rara vez & Nunca & N/A \\
\hline 17 & $\begin{array}{l}\text { Mi hijo(a) habla francés en la casa con los integrantes de la } \\
\text { familia. }\end{array}$ & & & & & \\
\hline 18 & $\begin{array}{l}\text { Mi hijo(a) lee (libros, revistas, cosas en internet, etc.) en } \\
\text { francés en la casa. }\end{array}$ & & & & & \\
\hline 19 & $\begin{array}{l}\text { Mi hijo(a) escucha música o videos (por internet o en la radio, } \\
\text { etc.) en francés en la casa. }\end{array}$ & & & & & \\
\hline 20 & Mi hijo(a) habla en francés con los profesores en la escuela. & & & & & \\
\hline 21 & Mi hijo(a) habla en francés con los compañeros en la escuela. & & & & & \\
\hline 22 & Mi hijo(a) lee (textos) en francés en la escuela. & & & & & \\
\hline 23 & Mi hijo(a) escribe en francés en la casa. & & & & & \\
\hline 24 & Mi hijo(a) escribe en francés en la escuela. & & & & & \\
\hline
\end{tabular}




\section{Motivación}

\begin{tabular}{|c|c|c|c|c|c|c|}
\hline & Indicador & Siempre & A veces & Rara vez & Nunca & N/A \\
\hline 28 & A mi hijo(a) le gusta llegar puntual a la escuela. & & & & & \\
\hline 29 & Mi hijo(a) hace las tareas de la escuela. & & & & & \\
\hline 33 & $\begin{array}{l}\text { Mi hijo(a) recuerda el nombre de la mayoría de sus compañeros } \\
\text { de curso. }\end{array}$ & & & & & \\
\hline 34 & $\begin{array}{l}\text { Cuando le pregunto a mi hijo(a) ¿Cómo te fue? ¿Qué hiciste } \\
\text { hoy en la escuela?, me contesta con interés sobre lo que hizo. }\end{array}$ & & & & & \\
\hline 36 & Mi hijo(a) me cuenta las cosas que aprende en la escuela. & & & & & \\
\hline 37 & $\begin{array}{l}\text { Mi hijo(a) participa en alguna actividad extra programática en } \\
\text { la escuela (taller de música, de arte, de deporte u otro). }\end{array}$ & & & & & \\
\hline 38 & $\begin{array}{l}\text { Mi hijo(a) saca libros de la biblioteca de la escuela por } \\
\text { iniciativa propia. }\end{array}$ & & & & & \\
\hline 39 & A mi hijo(a) le gusta hablar en español fuera de la escuela. & & & & & \\
\hline 40 & A mi hijo(a) le gusta estudiar español en la casa. & & & & & \\
\hline 45 & $\begin{array}{l}\text { A mi hijo(a) le gusta leer los libros de español que trae de la } \\
\text { biblioteca. }\end{array}$ & & & & & \\
\hline
\end{tabular}

\section{Relación con la escuela}

\begin{tabular}{|c|c|c|c|c|c|c|}
\hline & Indicador & Siempre & A veces & Rara vez & Nunca & N/A \\
\hline 46 & $\begin{array}{l}\text { Cuando voy a la reunión de apoderados o a entrevistas, los } \\
\text { profesores me cuentan cómo le va a mi hijo(a). }\end{array}$ & & & & & \\
\hline 47 & $\begin{array}{l}\text { Cuando voy a la escuela, los profesores me responden las } \\
\text { consultas que tengo sobre mi hijo(a). }\end{array}$ & & & & & \\
\hline 48 & $\begin{array}{l}\text { Cuando voy a la escuela los directivos, me responden las } \\
\text { consultas que tengo. }\end{array}$ & & & & & \\
\hline 49 & $\begin{array}{l}\text { En la escuela me han entendido cuando he tenido problemas } \\
\text { para asistir a las reuniones. }\end{array}$ & & & & & \\
\hline 50 & $\begin{array}{l}\text { A la escuela le gusta que que los padres participemos en las } \\
\text { actividades extracurriculares. }\end{array}$ & & & & & \\
\hline 51 & $\begin{array}{l}\text { A la escuela le gusta que conozcamos lo que ellos hacen con } \\
\text { nuestros hijos(as). }\end{array}$ & & & & & \\
\hline 52 & $\begin{array}{l}\text { Cuando voy a la escuela se dan el tiempo para explicarme } \\
\text { cómo va mi hijo, a pesar de que no manejo bien el español. }\end{array}$ & & & & & \\
\hline 53 & $\begin{array}{l}\text { Según mi hijo(a), sus profesores lo tratan igual que a los } \\
\text { estudiantes chilenos. }\end{array}$ & & & & & \\
\hline 54 & $\begin{array}{l}\text { Según mi hijo(a), los directivos lo tratan igual que a los } \\
\text { estudiantes chilenos. }\end{array}$ & & & & & \\
\hline 42 & A mi hijo le gusta enseñarme palabras en español. & & & & & \\
\hline 43 & $\begin{array}{l}\text { A mi hijo le gusta juntarse con compañeros que hablan español } \\
\text { fuera de la escuela. }\end{array}$ & & & & & \\
\hline
\end{tabular}

Muchas gracias por sus respuestas! 
Cuestionario CPAHPAL en Creole

\section{Etid sou pèsepsyon}

nan pratik alfabetizasyon ak lang Kesyonè pou paran ak responsab yo

Sondaj sa a se yon pati nan Pwojè Mèt la ki rele "Pèsepsyon paran oswa responsab elèv ki ap aprann Panyòl kòm dezyèm lang nan kad lekòl”.

Rezon ki fè sondaj sa a, se pou ka konnen sa'w panse etanke papa (manman) oswa responsab.

4. Sou lang, aktivite ki gen ladann li ak ekri nan lekòl ak lakay ou.

5. Motivasyon pitit ou pou lekòl ak Panyòl.

6. Relasyon ou ak lekòl.

Lide a se pataje pèsepsyon sa yo ak pwofesè yo ak otorite lekòl yo. Konsa, yo pral entegre vizyon nou pou pote chanjman miyò ak opòtinite y’ap ofri pou pitit ou aprann.

Nou remèsye'w paske ou reponn kesyonè sa a pi klè ke posib. Repons kesyonè a ak enfòmasyon pèsonèl yo konfidansyèl epi yo pral itilize yo sèlman pou objektif etid sa a.

Pou fè sa, tanpri swiv enstriksyon ki anba yo:

Tanpri, reponn tout kesyon ki nan kesyonè sa a.

D Li chak kesyon ak anpil atansyon epi make avèk yon iks (X) nan bwat ki koresponn ak repons ou an. Ou dwe toujou tcheke yon sèl bwat.

$>\quad \mathrm{Si}$ ou konfonn repons yo, ranpli tout bwat yo epi make yon nouvo iks (X) nan bwat ki kòrèk la.

$>\quad$ Lè yo mande pou'w ekri nimewo ak repons ki ekri yo, tanpri fè sa yon fason klè, egzak ak fasil pou li.

$>\quad$ Lè'w fin repon kesyon yo remèt kesyonè a bay moun ki responsab li a nan lekòl la.

$>\quad$ Dezyèm pati nan sondaj sa a gen ladann kesyon pou reponn tou kout.

¡Mèsi anpil dèske ou kowopere! 


\section{Enfòmasyon jeneral}

16. Non

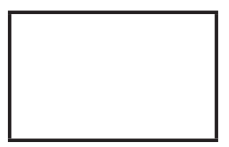

17. Siyati Papa

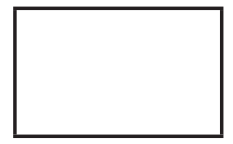

18. Siyati Manman

(si aplikab)

Sèks: Maskilen Feminen

19. Laj:

20. Kantite ane ou nan Chili:

21. Lang manman'w:

22. Lòt lang:

23. Nivo panyòl: (1) $\mathrm{Ba}$

(2)Pasab

(3)Avanse

(4)Siperyè

24. Ane lekòl ou fè:

25. Ki kote ou aprann panyòl?:

26. W'ap travay kounya?: Sí No

\section{Enfòmasyon lojman}

27. Nan ki komin w'ap viv?:

28. Ak konbyen moun w'ap viv kounya?:

29. Konbyen pitit ou genyen?:

Pou kontinye, tanpri mete timoun yo $\mathbf{k}$ ap viv avèk ou:

\begin{tabular}{|c|c|c|c|c|c|c|}
\hline Non & Siyati papa & $\begin{array}{l}\text { Siyati Manman } \\
\text { (si aplikab) }\end{array}$ & Sèks & Laj & Fèt chili & $\begin{array}{l}\text { Ki klas ane sa } \\
(2017)\end{array}$ \\
\hline & & & ${ }_{-}^{\mathrm{M}}{ }_{-} \mathrm{F}$ & & $\begin{array}{c}\mathrm{Wi} \\
\\
\text { Non }\end{array}$ & \\
\hline & & & ${ }_{-\mathrm{M}} \_\mathrm{F}$ & & $\begin{array}{c}\mathrm{Wi} \\
\\
\text { Non }\end{array}$ & \\
\hline & & & ${ }_{-}^{\mathrm{M}}{ }_{-}^{\mathrm{F}}$ & & $\begin{array}{c}\mathrm{Wi} \\
\mathrm{Non} \\
\end{array}$ & \\
\hline & & & ${ }_{-\mathrm{M}} \_\mathrm{F}$ & & $\begin{array}{c}\text { Wi } \\
\text { Non }\end{array}$ & \\
\hline & & & ${ }^{\mathrm{M}} \_\mathrm{F}$ & & $\begin{array}{c}\mathrm{Wi} \\
\mathrm{Non}\end{array}$ & \\
\hline
\end{tabular}




\section{Premye pati}

Sonje pou'w make ak yon iks $(\mathrm{X})$ opsyon ki pi apwopriye selon pèsepsyon ou. Si ou wè as yo di a pa gen rapò avè'w, chwazi Pa Aplikab (P/A).

\section{Itilizasyon lang yo}

\section{Pou Panyòl}

\begin{tabular}{|c|c|c|c|c|c|c|}
\hline & Endikatè & Toutan & Pafwa & Raman & Ditou & $\mathbf{P} / \mathbf{A}$ \\
\hline 1 & Pitit mwen an pale Panyòl lakay la avèk manm fanmi an. & & & & & \\
\hline 2 & $\begin{array}{l}\text { Pitit mwen an li (liv, magazin, bagay sou entènèt, etc.) an } \\
\text { Panyòl lakay la. }\end{array}$ & & & & & \\
\hline 3 & $\begin{array}{l}\text { Pitit mwen an tande mizik oubyen gade video (sou entenèt } \\
\text { oubyen nan radio, etc.) an Panyòl lakay la. }\end{array}$ & & & & & \\
\hline 4 & Pitit mwen an pale Panyòl avek pwofesè yo nan lekòl la. & & & & & \\
\hline 5 & Pitit mwen an pale Panyòl ak kamarad li yo nan lekòl la. & & & & & \\
\hline 6 & Pitit mwen an li (tèks) an Panyòl nan lekòl la. & & & & & \\
\hline 7 & Pitit mwen an ekri an Panyòl lakay la. & & & & & \\
\hline 8 & Pitit mwen an ekri an Panyòl nan lekòl la. & & & & & \\
\hline
\end{tabular}

\section{Pou Kreyòl}

\begin{tabular}{|c|c|c|c|c|c|c|}
\hline & Endikatè & Toutan & Pafwa & Raman & Ditou & P/A \\
\hline 9 & Pitit mwen an pale Kreyòl lakay la avèk fanmi an. & & & & & \\
\hline 10 & $\begin{array}{l}\text { Pitit mwen li (liv, magazin, bagay sou entènèt, etc.) an } \\
\text { Kreyòl lakay la. }\end{array}$ & & & & & \\
\hline 11 & $\begin{array}{l}\text { Pitit mwen an tande mizik ak gade video (sou entènèt } \\
\text { oubyen nan radio, etc.) an Kreyòl lakay la. }\end{array}$ & & & & & \\
\hline 12 & Pitit mwen an pale Kreyòl avèk pwofesè yo nan lekòl la. & & & & & \\
\hline 13 & Pitit mwen an pale Kreyòl ak kamarad li yo nan lekòl la. & & & & & \\
\hline 14 & Pitit mwen an li (tèks) an Kreyòl nan lekòl la & & & & & \\
\hline 15 & Pitit mwen an ekri an Kreyòl lakay la. & & & & & \\
\hline 16 & Pitit mwen an ekri an Kreyòl nan lekòl la. & & & & & \\
\hline
\end{tabular}


Estudios Pedagógicos XLV, $\mathrm{N}^{\circ}$ 3: 51-77, 2019

CUESTIONARIO DE APODERADOS DE ESTUDIANTES HAITIANOS SOBRE PRÁCTICAS DE ALFABETIZACIÓN Y

LENGUAS

Pou Fransè (Si ou pa pale Fransè, pa konplete nimewo sa yo epi kontinye nan nimewo 25 la)

\begin{tabular}{|c|c|c|c|c|c|c|}
\hline & Endikatè & Toutan & Pafwa & Raman & Ditou & P/A \\
\hline 17 & Pitit mwen an pale Fransè lakay la avèk fanmi an. & & & & & \\
\hline 18 & $\begin{array}{l}\text { Pitit mwen li (liv, magazin, bagay sou entènèt, etc.) an } \\
\text { Fransè lakay la }\end{array}$ & & & & & \\
\hline 19 & $\begin{array}{l}\text { Pitit mwen an tande mizik ak gade video (sou entènèt } \\
\text { oubyen nan radio, etc.) an Fransè lakay la. }\end{array}$ & & & & & \\
\hline 20 & Pitit mwen an pale Fransè avèk pwofesè yo nan lekòl la. & & & & & \\
\hline 21 & Pitit mwen an pale Fransè ak kamarad li yo nan lekòl la & & & & & \\
\hline 22 & Pitit mwen an li (tèks) an Fransè nan lekòl la & & & & & \\
\hline 23 & Pitit mwen an ekri an Fransè lakay la. & & & & & \\
\hline 24 & Pitit mwen an ekri an Fransè nan lekòl la. & & & & & \\
\hline
\end{tabular}

\section{Motivasyon pou lekòl la}

\begin{tabular}{|c|c|c|c|c|c|c|}
\hline & Endikatè & Toutan & Pafwa & Raman & Ditou & $\mathbf{P} / \mathbf{A}$ \\
\hline 28 & Pitit mwen an renmen rive alè nan lekòl la. & & & & & \\
\hline 29 & Pitit mwen an fè devwa lekòl la bay li. & & & & & \\
\hline 31 & Pitit mwen an patisipe nan aktivite nan lekòl la. & & & & & \\
\hline 33 & Pitit mwen an sonje non pi fò nan kamarad klas li yo. & & & & & \\
\hline 34 & $\begin{array}{l}\text { Lè mwen mande pitit mwen an: koman sa te ye pou ou? } \\
\text { ¿ kisa ou te fè lekòl la jodia?,li rakonte'm avèk enterè sa } \\
\text { li te fè.. }\end{array}$ & & & & & \\
\hline 36 & Pitit mwen an eksplike'm sa li te aprann nan lekòl la. & & & & & \\
\hline 37 & $\begin{array}{l}\text { Pitit mwen an patisipe nan kèk aktivite anplis nan lekòl la } \\
\text { (mizik, atis pent, atizana espò oswa lòt atelye). }\end{array}$ & & & & & \\
\hline 38 & $\begin{array}{l}\text { Pitit mwen an pran liv nan bibliyotèk lekòl la pou pwòp } \\
\text { inisyativ li }\end{array}$ & & & & & \\
\hline 39 & Pitit mwen an renmen pale Panyòl deyò lekòl la. & & & & & \\
\hline 40 & Pitit mwen an renmen etidye Panyòl lakay la. & & & & & \\
\hline 45 & Pitit mwen renmen li liv Panyòl li pran nan bibliyotèk la. & & & & & \\
\hline
\end{tabular}




\section{Relasyon a lekòl la}

\begin{tabular}{|c|c|c|c|c|c|c|}
\hline & Endikatè & Toutan & Pafwa & Raman & Ditou & $\mathbf{P} / \mathbf{A}$ \\
\hline 46 & $\begin{array}{l}\text { Lè mwen ale nan reyinyon paran oswa entèvyou, pwofesè } \\
\text { yo di m' ki jan pitit la ye ak lekòl la. }\end{array}$ & & & & & \\
\hline 47 & $\begin{array}{l}\text { Lè mwen ale lekòl la, pwofesè yo reponn kesyon mwen } \\
\text { genyen sou pitit mwen an. }\end{array}$ & & & & & \\
\hline 48 & $\begin{array}{l}\text { Lè mwen ale lekòl la, administratè yo reponn kesyon } \\
\text { mwen genyen yo. }\end{array}$ & & & & & \\
\hline 49 & $\begin{array}{l}\text { Nan lekòl la, yo konprann mwen lè mwen te gen pwoblem } \\
\text { pou mwen vini nan reyinyon. }\end{array}$ & & & & & \\
\hline 50 & $\begin{array}{l}\text { Lekòl la renmen nou menm paran yo patisipe nan aktivite } \\
\text { ki andeyò lekòl la. }\end{array}$ & & & & & \\
\hline 51 & $\begin{array}{l}\text { Lekòl la renmen lè nou konnen ki sa yo fè ak timoun nou } \\
\text { yo. }\end{array}$ & & & & & \\
\hline 52 & $\begin{array}{l}\text { Lè mwen ale lekòl la, yo ban'm tan pou esplike ki jan pitit } \\
\text { mwen ap fè, menmsi mwen pa pale Panyòl byen. }\end{array}$ & & & & & \\
\hline 53 & $\begin{array}{l}\text { Selon pitit mwen an, pwofesè li yo trete'l tankou elèv } \\
\text { Chilyen yo. }\end{array}$ & & & & & \\
\hline 54 & $\begin{array}{l}\text { Selon pitit mwen an, administratè yo trete'l tankou elèv } \\
\text { Chilyen yo. }\end{array}$ & & & & & \\
\hline 42 & Pitit mwen an renmen anseye'm mo Panyòl. & & & & & \\
\hline 43 & $\begin{array}{l}\text { Pitit mwen an renmen rankontre avèk kamarad k'ap pale } \\
\text { Panyòl deyò lekòl la. }\end{array}$ & & & & & \\
\hline
\end{tabular}

\section{¡Mèsi anpil pou repons ou yo!}


